\title{
Data-driven Security-Constrained AC-OPF for Operations and Markets
}

\author{
Halilbasic, Lejla; Thams, Florian; Venzke, Andreas; Chatzivasileiadis, Spyros; Pinson, Pierre
}

Published in:

Proceedings of 20th Power Systems Computation Conference

Link to article, DOI:

10.23919/PSCC.2018.8442786

Publication date:

2018

Document Version

Peer reviewed version

Link back to DTU Orbit

Citation $(A P A)$ :

Halilbasic, L., Thams, F., Venzke, A., Chatzivasileiadis, S., \& Pinson, P. (2018). Data-driven SecurityConstrained AC-OPF for Operations and Markets. In Proceedings of 20th Power Systems Computation Conference IEEE. https://doi.org/10.23919/PSCC.2018.8442786

\section{General rights}

Copyright and moral rights for the publications made accessible in the public portal are retained by the authors and/or other copyright owners and it is a condition of accessing publications that users recognise and abide by the legal requirements associated with these rights.

- Users may download and print one copy of any publication from the public portal for the purpose of private study or research.

- You may not further distribute the material or use it for any profit-making activity or commercial gain

- You may freely distribute the URL identifying the publication in the public portal 


\title{
Data-driven Security-Constrained AC-OPF for Operations and Markets
}

\author{
Lejla Halilbašić, Florian Thams, Andreas Venzke, Spyros Chatzivasileiadis, Pierre Pinson \\ Center for Electric Power and Energy \\ Technical University of Denmark \\ Kongens Lyngby, Denmark \\ \{lhal, fltha, andven, spchatz, ppin\}@elektro.dtu.dk
}

\begin{abstract}
In this paper, we propose a data-driven preventive security-constrained AC optimal power flow (SC-OPF), which ensures small-signal stability and N-1 security. Our approach can be used by both system and market operators for optimizing redispatch or AC based market-clearing auctions. We derive decision trees from large datasets of operating points, which capture all security requirements and allow to define tractable decision rules that are implemented in the SC-OPF using mixedinteger nonlinear programming (MINLP). We propose a secondorder cone relaxation for the non-convex MINLP, which allows us to translate the non-convex and possibly disjoint feasible space of secure system operation to a convex mixed-integer OPF formulation. Our case study shows that the proposed approach increases the feasible space represented in the SCOPF compared to conventional methods, can identify the global optimum as opposed to tested MINLP solvers and significantly reduces computation time due to a decreased problem size.
\end{abstract}

Index Terms-Security-constrained OPF, small-signal stability, convex relaxation, mixed-integer non-linear programming.

\section{INTRODUCTION}

The Security-Constrained Optimal Power Flow (SC-OPF) is an important tool for power system operation and planning in order to maintain power system security. The SC-OPF identifies optimal preventive control actions compliant with the $\mathrm{N}-1$ criterion, which ensure that the system can withstand the loss of any major component without violating system constraints. To this end, the constraint set of the conventional OPF is extended to include all considered contingency scenarios. The SC-OPF can be applied at different planning stages to either determine an optimal and $\mathrm{N}-1$ secure dispatch or to identify optimal redispatching actions closer to real-time. SC-OPFs are non-linear and non-convex problems with both continuous and discrete variables. A major drawback of conventional SC-OPF algorithms is the problem size and the negligence of stability requirements [1], which cannot be expressed as linear or nonlinear constraints and are therefore difficult to incorporate in a straightforward way. We address both challenges by using a data-driven approach to preventive SC-OPF, which is computationally tractable and based on decision trees that capture all $\mathrm{N}-1$ and small-signal stability considerations. We

This work is supported by the European project BEST PATHS, Grant No. 612748, and the ForskEL-project 12264 BestPaths for DK. extend our previous work in [2] to an AC-OPF context with the intention to bridge the gap between power system operation and markets. We incorporate the knowledge extracted from the data in the OPF using convex Mixed-Integer Programming (MIP) and relax the non-convex $\mathrm{AC}$ power flow equations through a Second Order Cone (SOC) relaxation. We further use the data to strengthen the relaxation. To the authors' knowledge, this is the first SC-OPF formulated as a MixedInteger Second Order Cone Program (MISOCP). Our approach combines the advantages of convex MIP and data analytics and allows for a fast online solution. It extends the feasible space in order to reflect the true non-convex area of operation and includes the true optimal solution.

Research in SC-OPF algorithms has primarly investigated the $\mathrm{N}-1$ security criterion and the reduction of the associated computational burden, while less focus has been put on stability requirements. However, only enforcing the $\mathrm{N}-1$ criterion does not necessarily guarantee stability and hence, feasiblity of the solution. In order to translate small-signal stability constraints to an OPF context, eigenvalue-sensitivities with respect to OPF decision variables are used in [3] and [4]. However, the required computational effort and the scalability of the proposed methods are still major challenges. In [5] hyperplanes are used to approximate the security boundary and are incorporated in a DC-OPF. Operating points outside the convex space constructed by the hyperplanes are neglected though, increasing the likelihood of missing the optimal solution.

The authors in [6] have identified the Security-Constrained AC-OPF as the "ultimate goal" for market software to be used by Independent System Operators (ISO) across the US. They outline the oversimplification of current software, often requiring operator intervention and resulting in suboptimal solutions due to inaccurate ISO models, which only use estimates for reactive power and voltage constraints. Improved modeling of voltage and stability constraints can lead to more realistic dispatch decisions and better market signals, resulting in up to $10 \%$ cost savings worldwide [7]. The relative amount of potential savings will increase over the course of the coming decades due to the ongoing electrification of the entire energy industry. Electricity market participants in the US are already saving over half a billion dollars annually as a result of the implementation of MIP in day-ahead and real-time markets 
[8]. Due to significant improvements since the 1990s, MIP has been widely applied accross various industries, increasing expertise, which only reinforces further development of MIP approaches.

SOCP has gained increased attention for power system applications as it is computationally less demanding than other relaxations, such as Semi-Definite Programming (SDP). Furthermore, it can be extended to mixed-integer problems, where the SDP relaxation fails even on small scales. MISOCP has been introduced for OPF problems incorporating optimal transmission switching and capacitor placement in [9] and [10]. The challenge in SOCP for OPF problems lies in convexifying the coupling contraint between voltage angles and SOC variables, which are introduced to remove the nonconvexities arising from the AC power flow equations. Several strengthening techniques have been proposed in [9] and [10], which significantly contribute to the advancement of MISOCP for real-life applications. However, these techniques still do not achieve to avoid optimality gaps and can be computationally demanding, negating the benefit of convex MIP solvers.

The main contribution of this work is a data-driven SCOPF algorithm formulated as a MISOCP, which (a) can be used by system operators as a security analysis tool (e.g., for redispatch), (b) can be used by market operators, approaching the "ultimate goal" for market software [6], (c) incorporates the N-1 security criterion, (d) ensures small-signal stability for the base case and all considered contingencies, (e) is scalable, (f) exploits the maturity of convex MIP solvers in order to identify the optimal operating point within a non-convex and possibly disjoint stability area, (g) can be solved fast, as all computation related to the small-signal stability assessment and $\mathrm{N}-1$ criterion is done offline and the OPF problem size is significantly decreased, (h) uses data to stregthen the relaxation, (i) can be solved to global optimality where tested non-convex solvers fail and (j) alleviates the need for iterative contingency analyses, which are currently used by system operators to define the boundaries of the secure system space.

The remainder of this paper is organized as follows: Section II describes the proposed methodology including the derivation of decision rules from data and focuses mainly on the incorporation of those rules in nonlinear and non-convex SCOPF algorithms. Section III presents results from a case study. Section IV concludes and gives an outlook on future work.

\section{METHOD}

Our approach consists of an offline security assessment, which includes the database generation and derivation of decision rules, and an online SC-OPF, which incorporates the decision rules ensuring power system security. The database generation, stability analysis and knowledge extraction have been discussed in more detail in our previous work [2] and we will only highlight the main features here. This work focuses mainly on the implementation of the decision rules in AC-OPF algorithms and applications of the proposed method.

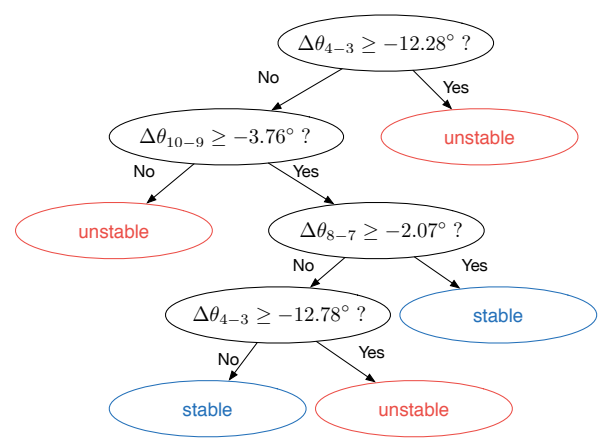

Figure 1. A simple illustrative decision tree.

\section{A. Offline Security Assessment}

A large database of operating points is created for various load levels based on AC power flow calculations. All possible generation patterns corresponding to the specified load levels are evaluated using specified step sizes to discretize the space of possible dispatch combinations. In order to perform the small-signal stability assessment of the simulated operating points, small-signal models are derived for the base case and all considered contingencies. A database is generated, which contains the operating points together with the information on whether they are stable or unstable. Challenges related to the computational demand of the database generation, the appropriate choice of discretization intervals and the determination of the stability boundary have been addressed in [2]. Ongoing work focuses on further improvements.

Once the database is generated, decision rules based on appropriate features, that need to be selected, can be derived for the OPF, which will lead the solution to a stable operating region. These features have to be easily accessible in an AC-OPF formulation to allow for a fast online computation. Voltage angle differences along transmission lines are explicitly modeled in both the security analysis and the AC-OPF, representing coupling variables. Among the tested features, they have proven to most efficiently capture all security requirements and are thus chosen as features for the knowledge extraction (i.e., the derivation of the decision rules). Additionally, if approximations of the OPF are used, which do not explicitly model voltage angles, angle differences can be translated to line capacity limits using appropriate mapping strategies as explained in [2]. We employ decision trees (DTs) as the knowledge extraction method, as they are comprehensible and easy to interpret for the operator. They are trained based on the selected features. Moreover, the conditional rules defined by the DT can be directly incorporated through binary variables in an optimization problem. A simple illustrative example is shown in Fig. 1.

We refer to our previous work for details on the smallsignal models, the DT generation including the reduction of mislassifications and the dependence of the DT accuracy on the database size/discretization [2]. 


\section{B. Preventive SC-OPF - Current Practice}

The traditional preventive SC-OPF (PSC-OPF) aims at finding the optimal least-cost dispatch ensuring $\mathrm{N}-1$ security. It extends the conventional nonlinear and non-convex ACOPF by including additional constraints to ensure feasibility of the identified solution after the occurrence of any specified contingency. The AC-OPF is given as follows:

$$
\begin{aligned}
& \min _{\mathbf{x}} \sum_{i=1}^{N_{G}} C_{i}\left(P_{i}^{G}\right), \\
& \text { s.t. } P_{i}^{G}-P_{i}^{D}=G_{i i} V_{i}^{2} \\
& \quad+\sum_{j=1, j \neq i}^{N_{B}} V_{i} V_{j}\left(G_{i j} \cos \left(\theta_{i j}\right)+B_{i j} \sin \left(\theta_{i j}\right)\right), \quad \forall i \in \mathcal{N} \\
& \quad Q_{i}^{G}-Q_{i}^{D}=-B_{i i} V_{i}^{2} \\
& \quad+\sum_{j=1, j \neq i}^{N_{B}} V_{i} V_{j}\left(G_{i j} \sin \left(\theta_{i j}\right)-B_{i j} \cos \left(\theta_{i j}\right)\right), \quad \forall i \in \mathcal{N}, \\
& \quad \underline{\mathbf{x}} \leq \mathbf{x} \leq \overline{\mathbf{x}}, \\
& \theta_{\text {ref }}=0,
\end{aligned}
$$

where $\mathbf{x}$ represents the set of optimization variables including active power generation $\mathbf{P}^{\mathrm{G}}$, reactive power generation $\mathbf{Q}^{\mathrm{G}}$, voltage magnitudes $\mathbf{V}$ and voltage angles $\theta . \mathcal{N}$ denotes the set of buses. The objective function (1) minimizes active power generation costs. Constraints (2) and (3) ensure active and reactive power balance at all nodes, where $G_{i j}$ and $B_{i j}$ represent the real and imaginary parts of the bus admittance matrix, respectively. Constraint (4) limits all decision variables within their upper and lower bounds. Equation (5) sets the voltage angle of the reference bus to zero. Line flow limits are not explicitly included here, as we derive limits from the security analysis, which will be introduced later.

Control variables (e.g., active and reactive power generation, transformer tap position etc.) in the PSC-OPF are not allowed to change from pre- to post-contingency states. The problem size of the OPF increases to $N_{c}+1$ compared to the conventional AC-OPF, where $N_{c}$ denotes the number of contingencies, rendering SC-OPFs computationally very demanding. The computational burden of SC-OPFs, especially for large systems with many considered contingencies, is a major challenge. Another challenging factor is the inclusion of system dynamics, i.e. stability considerations, in SC-OPF algorithms. Given that they cannot be incorporated as linear or nonlinear constraints in a straightforward way, common practice is to impose tighter constraints. Such conservative bounds on the feasible space should allow the system to return to a steady-state equilibrium after a fault event, but also lead to costly and suboptimal solutions.

Net Transfer Capacities (NTC) constitute one example of such conservatism. NTCs are maximum cross-zonal capacities available for trade on the day-ahead market, which are derived based on the $\mathrm{N}-1$ security criterion, accounting for

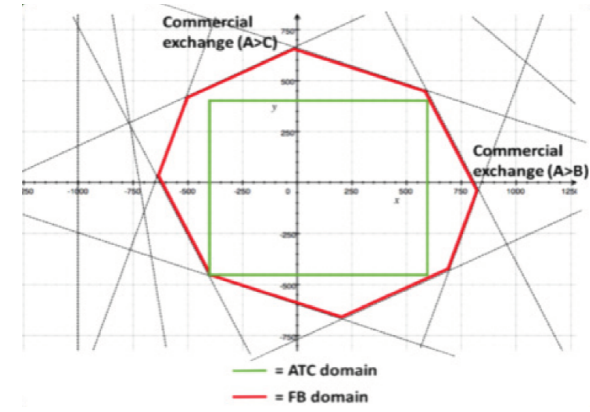

Figure 2. Security domain of Flow-Based Market Coupling (FBMC) and Available Transfer Capacity (ATC) [12]. ATCs are NTCs reduced by longterm capacity nominations.

thermal and stability limitations [11]. NTCs are fixed line flow limits, which are determined by transmission system operators (TSOs) in each direction of cross-border lines prior to the dayahead market-clearing. Improvements towards a more accurate representation of the physical reality of power systems have been achieved by introducing Flow-Based Market Coupling (FBMC), where a simplified European network representation is accounted for in the market-clearing. Critical branches $(\mathrm{CBs})$ inside of and between zones are identified, which could potentially be congested and limit the cross-border trade. All CBs are represented through Power Transfer Distribution Factors (PTDFs) and instead of assuming only one fixed NTC value for each cross-border line, all constraints imposed by the CBs are considered. Thus, a larger secure domain is offered to the market compared to static NTC approaches as depicted in Fig. 2. The market is then able to determine the optimal combination of commercial capacities between zones. Nonetheless, even flow based NTCs only capture one convex region of the entire feasible space and do not represent the non-convex reality of power system operations.

\section{Improving the Preventive SC-OPF Using Data}

To address the challenges described above, we propose a data-driven approach to SC-OPFs, which we introduced in previous work [2]. Non-convex and discontinous feasibility spaces are captured using decision trees, which define conditional constraints on appropriate decision variables, such as voltage angle differences $\theta_{i j}=\theta_{i}-\theta_{j}$ along transmission lines. Each branch $p$ of the DT contains a set of minimum and maximum voltage angle differences and leads to a different region of the feasibility space, one of which contains the leastcost, optimal solution. A visual illustration of our approach is depicted in Fig. 3. Instead of defining bounds that contain only one of the three stable regions depicted in the shades of blue, conditional rules allow to capture the entire feasible region within the red frame. The DT is incorporated in the SC-OPF using Mixed-Integer Programming (MIP). Each full branch is associated with a binary variable $y_{p}$, which if chosen, activates the corresponding upper and lower bounds on angle differences, or otherwise, leaves the original bounds unchanged: 


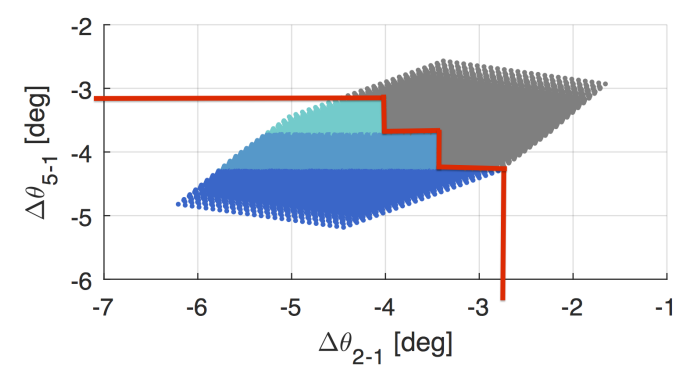

Figure 3. Illustrative example of non-convex space.

$$
\begin{array}{r}
y_{p} \cdot \underline{\theta_{i j, p}}-\left(1-y_{p}\right) \cdot \overline{\theta_{i j}} \leq \theta_{i j} \leq y_{p} \cdot \overline{\theta_{i j, p}}+\left(1-y_{p}\right) \cdot \overline{\theta_{i j}}, \\
\forall(i, j) \in \mathcal{L}, \forall p \in \mathcal{P} . \quad \text { (6) }
\end{array}
$$

$\mathcal{L}$ and $\mathcal{P}$ denote the set of lines and DT branches/paths, respectively. Only one DT branch, i.e. one set of minimum and maximum bounds on angle differences, can be chosen, which is imposed by adding constraint $\sum_{p \in \mathcal{P}} y_{p}=1$.

The data-driven SC-OPF is thus formulated as a MixedInteger Non-linear Program (MINLP), which is a NP-hard problem. One of the main contributions of this paper is that we relax the non-convex AC power flow equations using a Second Order Cone relaxation and obtain a SC-OPF formulated as a Mixed-Integer Second Order Cone Program (MISOCP), which is a convex mixed-integer program that can be solved efficiently by already existing solvers.

\section{Relaxing the Data-driven SC-OPF}

The SOC formulation of the OPF problem is based on [13], [14] and [9]. New variables are introduced to capture the nonlinearities and non-convexities of the $\mathrm{AC}$ power flow equations: (a) $u_{i}:=V_{i}^{2}$, (b) $c_{i j}:=V_{i} V_{j} \cos \left(\theta_{i j}\right)$ and (c) $s_{i j}:=-V_{i} V_{j} \sin \left(\theta_{i j}\right)$. The AC-OPF is transformed from the space of $\mathbf{x}:=\left\{\mathbf{P}^{\mathbf{G}}, \mathbf{Q}^{\mathbf{G}}, \mathbf{V}, \theta\right\}$ variables to the space of $\mathbf{X}_{\mathbf{S O C}}:=\left\{\mathbf{P}^{\mathrm{G}}, \mathbf{Q}^{\mathrm{G}}, \mathbf{u}, \mathbf{c}, \mathbf{s}\right\}$ variables and is given by:

$$
\begin{array}{rlr}
\min _{\mathbf{x} \text { SOC }} & \sum_{i=1}^{N_{G}} C_{i}\left(P_{i}^{G}\right) & \\
\text { s.t. } & P_{i}^{G}-P_{i}^{D}=G_{i i} u_{i} & \\
& +\sum_{j=1, j \neq i}^{N_{B}}\left(G_{i j} c_{i j}-B_{i j} s_{i j}\right), & \\
Q_{i}^{G}-Q_{i}^{D}=-B_{i i} u_{i} & \\
& -\sum_{j=1, j \neq i}^{N_{B}}\left(G_{i j} s_{i j}+B_{i j} c_{i j}\right), & \\
c_{i j}=c_{j i}, \quad s_{i j}=-s_{j i}, & \forall i \in \mathcal{N} \\
c_{i j}^{2}+s_{i j}^{2}=u_{i} u_{j}, & \forall(i, j) \in \mathcal{L} \\
& \underline{V_{i}^{2}} \leq u_{i} \leq \bar{V}_{i}^{2}, & \forall i \in \mathcal{N} .
\end{array}
$$

Active and reactive power generation are constrained by their usual limits and are not explicitly mentioned. The non-convex quadratic equality constraint (11) is relaxed through a secondorder cone constraint: $c_{i j}^{2}+s_{i j}^{2} \leq u_{i} u_{j}$. The SOC formulation of the OPF problem (7)-(12) is exact for radial networks and the optimal voltage angles can be recovered by solving:

$$
\theta_{i}-\theta_{j}=\operatorname{atan} 2\left(s_{j i}^{*}, c_{i j}^{*}\right), \quad \forall(i, j) \in \mathcal{L},
$$

where $*$ indicates the optimal solution obtained from the SOC-OPF. For meshed networks the above formulation is a strict relaxation, potentially resulting in solutions, which are infeasible for the original AC-OPF [9]. Reintroducing voltage angle variables and constraint (13) in problem (7)-(12) would render the SOC formulation exact for meshed networks, but also non-convex. Various convex approximations of constraint (13) have been proposed [9], [13]. In this paper we use a sequential conic procedure as proposed in [13], where the arctan function is linearized using a Taylor series expansion.

Conditional bounds on voltage angles are introduced using binary variables as described in Section II-C. Known limits on angle differences can also be used to define bounds on the SOC variables $\mathbf{c}$ and $\mathbf{s}$ :

$$
\begin{array}{r}
y_{p} \cdot \tan \left(\underline{\theta_{i j, p}}\right) \cdot c_{i j}-\left(1-y_{p}\right) \cdot \tan \left(\overline{\theta_{i j}}\right) \cdot c_{i j} \leq s_{j i}, \\
\forall(i, j) \in \mathcal{L}, \forall p \in \mathcal{P}, \\
y_{p} \cdot \tan \left(\overline{\theta_{i j, p}}\right) \cdot c_{i j}+\left(1-y_{p}\right) \cdot \tan \left(\overline{\theta_{i j}}\right) \cdot c_{i j} \geq s_{j i}, \\
\forall(i, j) \in \mathcal{L}, \forall p \in \mathcal{P} .
\end{array}
$$

Otherwise, the SOC variables $c_{i j}$ and $s_{i j}$ are only constrained by their implied upper and lower bounds $\pm \overline{V_{i} V_{j}}$, which in practice can be very loose. In order to tighten the relaxation, we determine the values for $c_{i j}$ and $s_{i j}$ along all lines $(i, j)$ for each operating point in the generated database and extract their minimum and maximum values to obtain tighter variable bounds.

\section{CASE Study}

We evaluate the performance of the proposed data-driven SC-OPF ensuring small-signal stability and $\mathrm{N}-1$ security on a modified version of the IEEE 14 bus test system [15]. Our case study consists of three parts. First, we compare the convex MISOCP formulation of the algorithm to a data-driven MINLP implementation and a standard (i.e., not data-driven) preventive SC-OPF. Its low computational effort makes the proposed MISOCP suitable for AC based market-clearing auctions as envisaged in the US. In Europe, however, electricity markets will probably continue to rely on DC approximations. To this end, we show how the proposed method can also be used by TSOs for optimizing redispatching actions in order to ensure $\mathrm{N}-1$ security and small-signal stability after the marketclearing. Finally, we analyze how the data-driven approach coupled with MIP notably extends the feasible space of the OPF.

The network data is given in [16] with some modifications introduced in [2]. Note that additionally the voltage setpoints of generators 4 and 5 are changed to 1.02 p.u. and 1.01 p.u., respectively, and the reactive power of generator 1 is limited within \pm 990 Mvar. The considered contingencies include all line and bus faults, except for faults at buses 1, 
2 and 6. These would lead to either instability/AVR limit violations or operating points, which are unstable for the remaining contingencies. Note that the standard PSC-OPF does not incorporate stability considerations, but only extends the constraint set to account for the defined $\mathrm{N}-1$ contingencies. Bus 1 with generator $g_{1}$ has been chosen as the slack bus for the base case and all N-1 cases. A step size of $0.5 \mathrm{MW}$ was chosen for discretizing the possible operating range and generating the database of operating points used for deriving the decision tree. See [2] for a comparison of test cases with different discretization intervals. The minimum damping ratio of an operating point over the base case and all $\mathrm{N}-1$ cases is used as a metric for small-signal stability. As usual in power system operation, we define a security margin by requiring a minimum damping ratio of 3\%. The derived DT contains 136 leaf nodes, out of which 74 point to operating regions, which are small-signal stable for the base case and all considered contingencies. Thus, 74 binary variables are used to define conditional constraints on voltage angle differences along lines and incorporate the stable regions in the data-driven SC-OPF. Note that 1.34 million operating points have been evaluated for this case study, from which more than 88000 fulfill the required minimum damping ratio. We derive the DT based on three different load levels (base case, $\pm 20 \%$ ). The data-driven SC-OPF was performed for all three levels seperately. Given that they showed very similar results, we present our results for the base case only. The voltage magnitude at generator buses is set to the corresponding generator voltage setpoint in order to ensure an appropriate voltage profile, which is fundamental to a secure system operation.

The small-signal analysis was performed using Matlab and Matpower 6.0 [16]. The MISOCP was implemented in Python using the Gurobi Optimizer. The standard PSC-OPF (i.e., NLP) and the MINLP implementation of the data-driven SCOPF were carried out in GAMS. BONMIN and COUENNE were used for solving the MINLPs, while CONOPT was used for the NLP.

\section{A. Comparison of Preventive and Data-driven SC-OPFs}

Table I lists the results of all three SC-OPF implementations. It can be observed that all implementations based on decision trees result in operating points, which exhibit minimum damping ratios that are non-negative and are thus stable for the base case and all considered N-1 contingencies. The solution of the standard PSC-OPF however is unstable for a fault at bus 5 and line 1-2, respectively. Thus, the identified preventive control actions do not fullfill the system's security requirements and would call for redispatching measures. As the PSC-OPF results in an operating point, which does not reflect the small-signal stability requirements, its total cost is lower than the other solutions. All three data-driven implementations result in different leaf nodes of the DT and thus in different regions of the feasible space with various degrees of conservatism. Higher damping ratios are also reflected by higher costs. All solutions include a stability margin, but only the most conservative result achieves a minimum damping ratio of more than $3 \%$. This can

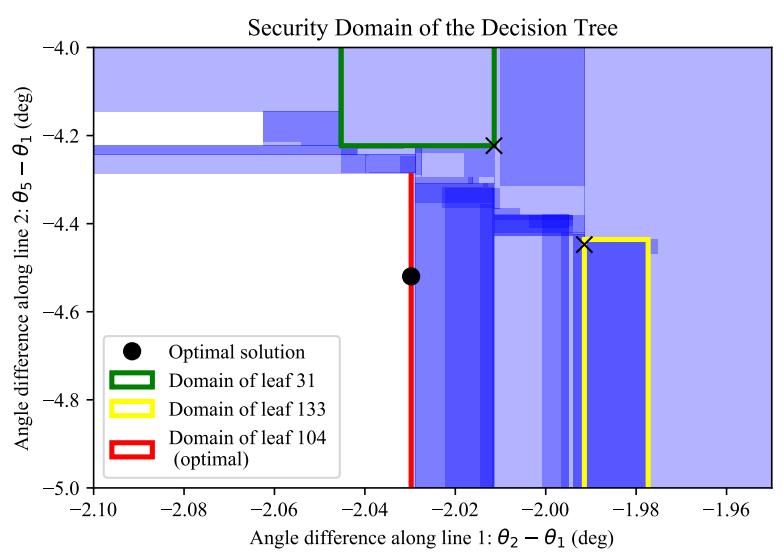

Figure 4. Non-convex security domain of the DT as a function of the angle differences along lines 1 and 2 . The blue shaded areas represent the stable regions defined by all DT branches. The darker the color, the more domains of different DT branches overlap. Note that the actual security domains covered by the different leaf nodes do not overlap and might even be disjoint, which however cannot be depicted by only two dimensions.

be explained by the fact that (a) the OPF is not limited to the specified discretization intervals of $0.5 \mathrm{MW}$, as used for the database generation and (b) DTs are not 100\% accurate leading to missclassifications. For cases, where the minimum damping ratio is a hard constraint, higher values can be required when deriving the DT in order to avoid violations. Fig. 4 illustrates the stable domain covered by all DT leaf nodes as a function of the angle differences along lines 1 and 2 . The rectangles indicate the domains covered by the three different leaf nodes obtained from the data-driven SC-OPF implementations with the location of the corresponding solution pointed out. It can be seen that the stable domain is non-convex and that safer operating points exhibiting higher damping ratios are located further away from the stability boundary.

The known scalability issues of COUENNE [10], which is a global optimization solver, lead to significantly higher execution times compared to BONMIN, which employs heurisitic methods for non-convex MINLPs. Also, the default settings for both solvers include a relative tolerance gap of $10 \%$ terminating the computation as soon as a solution is found, which lies within $10 \%$ of the true objective value. This explains the choice of different leaf nodes. However, even reducing the tolerance value does not change the outcome indicating that neither of the solvers is able to provide the best integer solution. The MISOCP is able to find the optimal integer solution, which has been verified by manually imposing the bounds defined by each DT branch. Specifically, we solved 74 AC-OPFs consecutively, one for each DT branch and imposed the corresponding bounds in order to determine the DT branch, which contains the least-cost, optimal NLP solution. Leaf node 104 actually contains the bounds, which result in the least-cost NLP solution. Notably, in this test case, the MISOCP relaxation is exact, i.e. its solution is feasible to the nonlinear, non-convex AC-OPF formulation and corresponds to the global optimum of the non-convex problem. 
TABLE I

RESULTS OF STANDARD PSC-OPF AND DATA-DRIVEN SC-OPF IMPLEMENTED AS MINLP AND MISOCP.

\begin{tabular}{|c|c|c|c|c|c|}
\hline & & \multirow{3}{*}{$\frac{\text { Standard PSC-OPF }}{\text { NLP }}$} & \multicolumn{3}{|c|}{ Data-driven SC-OPF } \\
\hline & & & \multicolumn{2}{|c|}{ MINLP } & \multirow{2}{*}{ MISOCP } \\
\hline & & & COUENNE & BONMIN & \\
\hline Cost & $(€ / h)$ & 3022.83 & 3445.09 & 3381.21 & 3365.95 \\
\hline Runtime & (s) & 10.06 & 1000.16 & 96.16 & 5.58 \\
\hline Iterations* & $(-)$ & - & - & - & 4 \\
\hline Min. damping ratio & $(\%)$ & unstable & 3.27 & 2.97 & 2.53 \\
\hline Leaf node & $(-)$ & - & 31 & 133 & 104 \\
\hline
\end{tabular}

Nonetheless, given that the MISOCP formulation is still a relaxation, identified solutions can be infeasible for the original problem. Therefore, we propose to use the MISOCP primarily to identify the optimal DT branch and hence the linear bounds on the feasible space, such that the optimal solution is included. Once these are known and the feasible space has been reduced, the integer variables can be omitted and a common AC-OPF (i.e., NLP) with the previously identified bounds on angle differences imposed as linear constraints can be performed to identify feasible and secure preventive control actions. The MISOCP solution serves as a lower bound and can validate the optimality of the AC-OPF solution.

Table I also outlines the savings in online computation time of the proposed SC-OPF method. The data-driven approach outperforms the usual PSC-OPF by more than $40 \%$, while at the same time incorporating more security requirements. Naturally, the computation time depends on the size of the DT and the number of binary variables used to represent it. Even though introducing binaries increases the OPF complexity, this side effect is offset by a significantly reduced overall size of the SC-OPF. While the PSC-OPF increases 32-fold when considering 31 contingencies, the proposed data-driven approach increases less than 4-fold for the same amount of contingencies. As indicated by constraint (6), $\left(2 \times N_{L} \times\right.$ $\left.N_{P}+1\right)$ additional linear inequality constraints are added, where $N_{L}$ and $N_{P}$ denote the number of network lines and DT paths, respectively. Hence, the complexity of the resulting MISOCP is primarily determined by the $N_{L}$ SOC constraints $c_{i j}^{2}+s_{i j}^{2} \leq u_{i} u_{j}$ and the $N_{P}$ binary variables.

Furthermore, given that the problem is a convex MIP, which can be solved efficiently by already existing solvers that are able to handle up to a few thousand binaries, the amount of discrete variables is not expected to be an obstacle. While solvers for MISOCPs have not yet reached the maturity of MILP solvers, recent work has demonstrated how general convex MIPs can be solved to global optimality by a sequence of MILPs using polyhedral outer approximations and continuous convex programs [17]. Large-scale instances of a similar type of problem class with up to 3000 binary variables and quadratic constraints have successfully been solved in [18] using state-of-the-art solvers and employing heuristic methods. The size of the DT is directly determined by the size of the dataset, which it is derived from. Hence, a more efficient method to generate the database of operating points, appropriate pruning of the DT and techniques to decompose the data-driven optimization are aspects that require a deeper

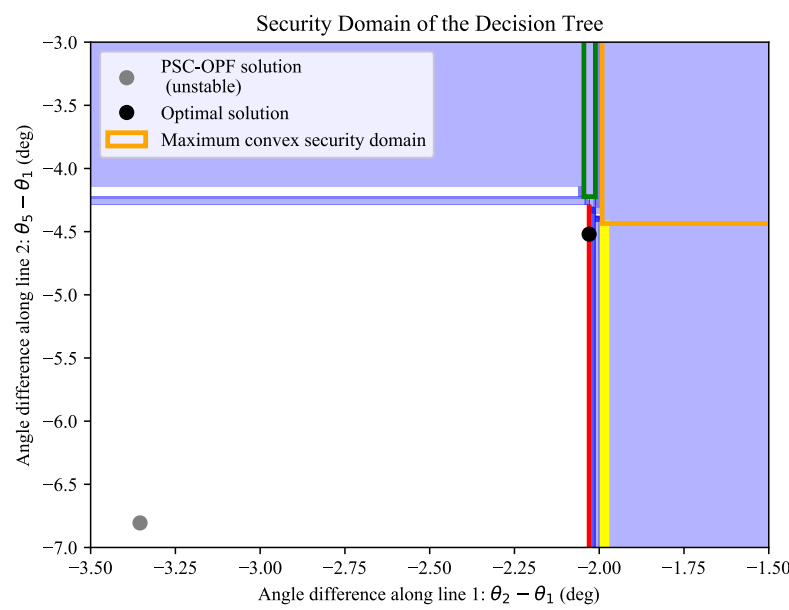

Figure 5. Visualization of redispatch and maximum convex security domain, which can be covered by one leaf node only.

analysis and are promising approaches to enhance the scalability performance of the method. One approach already used in the industry would be to focus only on the most critical $\mathrm{N}-1$ contingencies and angle differences.

\section{B. Optimizing Redispatch}

The method can be used by TSOs to identify optimal redispatching actions, where for example the market-based day-ahead dispatch, if identified as unstable, is redirected through the DT to a secure domain. We have reformulated the MISOCP in order to optimize redispatching actions, assuming that the costs of generator up- and down-regulation correspond to its marginal cost. Generators are assumed to be paid for up-regulation and charged for savings resulting from downregulation. We have used the unstable solution of the standard PSC-OPF listed in Table I as an input to the redispatch and successfully shifted the operating point to leaf node 104 . The cost of redispatch amounts to $343.12 €$, which corresponds to the original difference in objective values between the PSCOPF and MISOCP. The MISOCP, if implemented at the market stage, could result in more than $10 \%$ cost savings for the system operator and better reflects the locational impact of stability requirements.

\section{Enlarging the Feasible Space}

The generated database contains 1.34 million operating points, out of which $6.57 \%$ fulfill the $3 \%$ minimum damping 
ratio and define the feasible space of operation. We have evaluated how much of the entire stable region is covered by each individual leaf node, i.e. how many operating points fulfill the constraints imposed by the corresponding DT branch. All leaf nodes cover in total $99.91 \%$ of the considered stability region, where leaf node 7 accounts for most of it, capturing $77.78 \%$ of the entire feasible space. The implied bounds of leaf node 7 are depicted in Fig. 5. If we had considered the stability requirements in the OPF only by imposing bounds, which define a convex space, as it is currently done in practice, we would have been able to capture at most $77.78 \%$ of the feasible space, thereby also missing the optimal solution as can be seen in Fig. 5. Thus, our approach to incorporate the information provided by the database in the optimization problem through binary variables allows us to enlarge the feasible space by more than $28 \%$, enclosing the remaining $22.13 \%$, which are covered by the DT.

\section{COnclusion And Future Work}

In this paper we propose a data-driven approach to preventive SC-OPF, which ensures N-1 security and small-signal stability and uses decision trees to capture the non-convex and possibly disjoint space of stable operating points. We propose a new approach to translate data, which operators usually already have available from dynamic simulations, and include them in a simple way in an optimization framework. Binary variables are used to represent the knowledge extracted from the data giving rise to two levels of non-convexities: one that refers to the integrality of the binary variables and another one that concerns the $\mathrm{AC}$ power flow equations. We eliminate the latter using a SOC relaxation and obtain a convex MISOCP. MISOCPs generalize convex mixed-integer quadratic programs, for which solvers have already reached a high level of maturity. The proposed method reduces the overall problem size by avoiding to explicitly include all considered contingencies and incorporates stability requirements, both of which have been major challenges in SC-OPFs. Finally, we have shown that by using data coupled with MIP in (SC-)OPFs we can enlarge the feasible space represented in the OPF by more than $28 \%$.

The relaxed data-driven SC-OPF can be used to determine the optimal bounds (i.e., the optimal DT branch) on the feasible space, such that secure preventive control actions are ensured. Considering that the DT represents a non-convex operating space, identified preventive actions can be less conservative than those determined by current approaches. The need for iterative contingency analyses to identify suitable control actions is alleviated. The MISOCP formulation of the SC-OPF can be used by market operators as a marketclearing tool due to its low online computational effort but also by TSOs to determine optimal redispatching actions and the bounds on angle differences, which allow accommodating the redispatch.

In future work, we are planning to: (a) include uncertainty and corrective control policies for relaxed OPFs, which we developed in [19], (b) exploit the decomposable structure of the MISOCP to separate discrete and continuous variables and apply decomposition techniques with convergence guarantees resulting from the convex problem properties, (c) improve the database generation and determination of the stability boundary, which directly determine the DT size and (d) apply the method to larger systems.

\section{REFERENCES}

[1] F. Capitanescu, J. M. Ramos, P. Panciatici, D. Kirschen, A. M. Marcolini, L. Platbrood, and L. Wehenkel, "State-of-the-art, challenges, and future trends in security constrained optimal power flow," Electric Power Systems Research, vol. 81, no. 8, pp. 1731 - 1741, 2011.

[2] F. Thams, L. Halilbašić, P. Pinson, S. Chatzivasileiadis, and R. Eriksson, "Data-Driven Security-Constrained OPF," 10th IREP Symposium - Bulk Power Systems Dynamics and Control, 2017.

[3] R. Zárate-Miñano, F. Milano, and A. J. Conejo, "An OPF Methodology to Ensure Small-Signal Stability," IEEE Transactions on Power Systems, vol. 26, no. 3, pp. 1050-1061, 2011.

[4] J. E. Condren and T. W. Gedra, "Expected-Security-Cost Optimal Power Flow With Small-Signal Stability Constraints," IEEE Transactions on Power Systems, vol. 21, no. 4, pp. 1736-1743, 2006.

[5] M. Chávez-Lugo, C. R. Fuerte-Esquivel, C. A. Canizares, and V. J. Gutierrez-Martinez, "Practical Security Boundary-Constrained DC Optimal Power Flow for Electricity Markets," IEEE Transactions on Power Systems, vol. 31, no. 5, pp. 3358-3368, Sept 2016.

[6] M. B. Cain, R. P. O'Neill, and A. Castillo, "History of Optimal Power Flow and Formulations - Optimal Power Flow Paper 1," 2012 [Online]. Available: https://www.ferc.gov/industries/electric/indusact/market-planning/opf-papers/acopf-1-history-formulation-testing.pdf

[7] FERC Joint Boards on Security Constrained Economic Dispatch, "Study and Recommendations Regarding Security Constrained Economic Dispatch (SCED) in the Northeast by the Joint Board on Economic Dispatch for the Northeast Region," 2006. [Online]. Available: https://www.ferc.gov/industries/electric/indusact/joint-boards/south-recom.pdf

[8] FERC Staff, "Recent ISO Software Enhancements and Future Modeling Plans," 2011. [Online]. Available: https://www.ferc.gov/industries/electric/indus-act/rto/rto-iso-soft2011.pdf

[9] B. Kocuk, S. S. Dey, and X. Sun, "New Formulation and Strong MISOCP Relaxations for AC Optimal Transmission Switching Problem," IEEE Transactions on Power Systems, 2017, (in press).

[10] H. Hijazi, C. Coffrin, and P. V. Hentenryck, "Convex Quadratic Relaxations for Mixed-Integer Nonlinear Programs in Power Systems," NICTA, Tech. Rep., 2013.

[11] ENTSO-E, "Principles for Determining the Transfer Capacities in the Nordic Power Market," 2017.

[12] KU Leuven Energy Institute, "EI Fact Sheet: Cross-border Electrcity Trading: Towards Flow-based Market Coupling," 2015. [Online]. Available: http://set.kuleuven.be/ei/factsheets

[13] R. A. Jabr, "A Conic Quadratic Format for the Load Flow Equations of Meshed Networks," IEEE Transactions on Power Systems, vol. 22, no. 4, pp. 2285-2286, Nov 2007.

[14] A. G. Esposito and E. R. Ramos, "Reliable Load Flow Technique for Radial Distribution Networks," IEEE Transactions on Power Systems, vol. 14, no. 3, pp. 1063-1069, Aug 1999.

[15] S. Kodsi and C. Canizares, "Modeling and Simulation of IEEE 14 Bus System with FACTS Controllers," University of Waterloo, Waterloo, Canada, Tech. Rep., 2003.

[16] R. D. Zimmerman, C. E. Murillo-Sanchez, and R. J. Thomas, "MATPOWER: Steady-State Operations, Planning, and Analysis Tools for Power Systems Research and Education," IEEE Transactions on Power Systems, vol. 26, no. 1, pp. 12-19, Feb 2011.

[17] M. Lubin, E. Yamangil, R. Bent, and J. P. Vielma, "Polyhedral approximation in mixed-integer convex optimization," ArXiv e-prints, Jul. 2016.

[18] A. Bley, A. M. Gleixner, T. Koch, and S. Vigerske, "Comparing MIQCP Solvers to a Specialised Algorithm for Mine Production Scheduling," in Modeling, Simulation and Optimization of Complex Processes. Berlin, Heidelberg: Springer Berlin Heidelberg, 2012, pp. 25-39.

[19] A. Venzke, L. Halilbasic, U. Markovic, G. Hug, and S. Chatzivasileiadis, "Convex Relaxations of Chance Constrained AC Optimal Power Flow," IEEE Transactions on Power Systems, 2017, (in press). 